\title{
Vitex agnus-castus Extracts for Female Reproductive Disorders: A Systematic Review of Clinical Trials
}

Authors

Affiliations
M. Diana van Die ${ }^{1}$, Henry G. Burger ${ }^{2}$, Helena J. Teede ${ }^{3}$, Kerry M. Bone ${ }^{4}$

1 Royal Melbourne Institute of Technology-University, Bundoora, Victoria, Australia

2 Prince Henry's Institute of Medical Research, Clayton, Victoria, Australia

${ }^{3}$ Monash University, Clayton; Southern Health, Dandenong, Victoria, Australia

${ }^{4}$ University of New England, Armidale, New South Wales; MediHerb/Integria, Warwick, Queensland, Australia

\section{Key words \\ - Vitex agnus-castus \\ - Verbenaceae \\ premenstrual \\ - mastalgia \\ - hyperprolactinaemia \\ - systematic review}

received August 10, 2012 revised Sept. 9, 2012

accepted Sept. 17, 2012

Bibliography

Dol http://dx.doi.org/

10.1055/s-0032-1327831

Published online November 7 , 2012

Planta Med 2013; 79: 562-575

(c) Georg Thieme Verlag KG

Stuttgart · New York .

ISSN 0032-0943

Correspondence

Dr. Diana van Die

School of Health Sciences

RMIT University

PO Box 71

Bundoora, Victoria 3083

Australia

Phone: + 61403011151

Fax: + 61 (3) 99256539

diana.vandie@rmit.edu.au

\section{Abstract \\ $\nabla$}

Vitex agnus-castus L. (chaste tree; chasteberry) is a popular herbal treatment, predominantly used for a range of female reproductive conditions in Anglo-American and European practice. The objective of this systematic review was to evaluate the evidence for the efficacy and safety of Vitex extracts from randomised, controlled trials investigating women's health.

Eight databases were searched using Latin and common names for Vitex and phytotherapeutic preparations of the herb as a sole agent, together with filters for randomised, controlled trials or clinical trials. Methodological quality was assessed according to the Cochrane risk of bias and Jadad scales, as well as the proposed elaboration of CONSORT for reporting trials on herbal interventions.

Thirteen randomised, controlled trials were identified and twelve are included in this review, of which eight investigated premenstrual syndrome, two premenstrual dysphoric disorder, and two latent hyperprolactinaemia. For premenstrual syndrome, seven of eight trials found Vitex extracts to be superior to placebo ( 5 of 6 studies), pyridoxine

\section{Introduction}

$\nabla$

Use of complementary medicines is becoming increasingly prevalent in Western cultures, with women constituting the largest user group [1] Treatments for female reproductive health are often sought $[2,3]$. In this context, the phytotherapeutic agent Vitex agnus-castus fruit (chaste tree, chasteberry; family: Verbenaceae) is commonly employed for a range of female reproductive disorders [4], including premenstrual syndrome (PMS) and associated cyclic mastalgia, premenstrual dysphoric disorder (PMDD), lactation diffi-
(1), and magnesium oxide (1). In premenstrual dysphoric disorder, one study reported Vitex to be equivalent to fluoxetine, while in the other, fluoxetine outperformed Vitex. In latent hyperprolactinaemia, one trial reported it to be superior to placebo for reducing TRH-stimulated prolactin secretion, normalising a shortened luteal phase, increasing mid-luteal progesterone and $17 \beta$-oestradiol levels, while the other found Vitex comparable to bromocriptine for reducing serum prolactin levels and ameliorating cyclic mastalgia. Adverse events with Vitex were mild and generally infrequent. The methodological quality of the included studies varied, but was generally moderate-to-high. Limitations include small sample sizes in some studies, heterogeneity of conditions being treated, and a range of reference treatments.

Despite some methodological limitations, the results from randomised, controlled trials to date suggest benefits for Vitex extracts in the treatment of premenstrual syndrome, premenstrual dysphoric disorder and latent hyperprolactinaemia. Further research is recommended, and greater transparency in reporting for future trials.

culties, low fertility [5], and menopause-related complaints $[4,6]$.

PMS affects up to $40 \%$ women $[7,8]$, with a further $3-8 \%$ (or even $13-18 \%$ ) qualifying for the diagnosis of the more severe PMDD $[9,10]$. Cyclical breast symptoms are experienced by approximately $70 \%$ of women, while $22 \%$ experience moderate-to-extreme discomfort classified as cyclical mastalgia [11]. All of these common problems can be severe enough to interfere with usual activities. Conventional treatments such as hormonal interventions and synthetic antidepressant agents are not preferred options for a number of women. In this context, women often turn 
to complementary medicines for these and other reproductive health concerns.

Whilst these conditions involve different hormonal pathophysiologies, commonalities across some of them relate to prolactin elevation, proposed to be involved in premenstrual symptoms as well as in lactation difficulties. Although the exact cause has not yet been fully established, there is some evidence that premenstrual symptoms can be accompanied by a latent hyperprolactinaemia (LHP) $[12,13]$, the prolactin elevation occurring either premenstrually or in response to stressful situations $[14,15]$. Increased prolactin levels might inhibit corpus luteal development, thereby indirectly reducing the secretion of progesterone in the luteal phase of the menstrual cycle [16]. LHP has been associated with premenstrual mastalgia, benign breast cysts, and infertility $[12,17,18]$.

Vitex agnus-castus extracts may affect these conditions through dopaminergic activity via binding to dopamine-2 (DA-2) receptors [17], resulting in prolactin inhibition. (The pharmacology of Vitex is further reviewed below). Phytochemically, Vitex has been shown to contain dopaminergic compounds belonging to the diterpenes, as well as essential oil, flavonoids, and iridoid glycosides. The dried fruits are the most commonly used medicinal form and have a peppery taste. Today, Vitex is available in a range of pharmaceutical forms, including tinctures, fluid extracts, tablets, and homoeopathic preparations, and is commonly used throughout Europe and the English-speaking world.

In view of the clinical interest in Vitex for female reproductive disorders, the objective of this current review was to identify and systematically review all the data generated from randomised, controlled trials (RCTs) on the efficacy of Vitex agnus-castus in these conditions. Many of the earlier clinical studies on Vitex, such as those focussing on its galactagogue activity $[19,20]$, were open-label or observational studies. While certainly of interest, studies lacking a placebo control have obvious limitations, particularly for conditions susceptible to a substantial placebo response.

\section{Methods}

$\nabla$

Search strategy

The following electronic databases were searched (earliest to 2012): Medline, PubMed, EMBASE, The Cochrane Library, CINAHL, Ovid, Google scholar, and Web of Science.

Search terms used were: Vitex OR agnus-castus OR agnicasti OR chaste tree OR chasteberry OR monk's pepper OR hemp tree OR agneau chaste OR gatillier OR Keuschlamm OR kyskhedstrae OR agnuscasti OR Agnolyt OR Agnufemil OR Castufemin OR Cefanorm OR Femicur OR Gynocastus OR Hewekliman OR Kytta-femin OR Strotan OR agnomen AND randomised, controlled trial (where database search terms permitted).

In addition, further relevant papers were identified using the "related articles" function in PubMed, and by hand-searching reference lists of relevant journal articles and textbooks.

Where possible, authors were contacted [21-24] for further details of results, if these had not been reported as means and standard deviations.

\section{Study selection}

The search was restricted to randomised, controlled trials investigating extracts of Vitex agnus-castus in female reproductive conditions. All randomised, controlled trials, including cross-over trials, of Vitex versus placebo or a comparator treatment were included. Data from studies investigating multicomponent herbal formulations and homœopathic preparations were excluded. No language restrictions were imposed.

\section{Data extraction and quality assessment}

Details of trial design, duration and setting, condition under investigation, sample size, participants, outcome measures, adverse events, results, and methodological quality were extracted. Included studies were reviewed by two investigators (DvD and $\mathrm{KB}$ or $\mathrm{HB}$ ). To ascertain the validity of eligible randomised trials, two reviewers (DvD and KB) worked independently to determine selection bias, attrition bias, detection bias, performance bias, and reporting bias, using the Cochrane risk of bias assessment tool, RevMan [25]. The Jadad scale [26] was used to assess randomisation, double blinding, and reporting of withdrawals and dropouts. To supplement the quality assessment, additional criteria were assessed according to the proposed elaboration of the CONSORT checklist item 4 for reporting randomised, controlled trials of herbal medicines [27]. Disagreements were resolved by discussion between the two reviewers. If no agreement could be reached, it was decided that a third author would be consulted.

\section{Data analysis}

Quantitative meta-analysis was performed where possible, for independent studies investigating a common disorder, with a common comparator, compatible endpoints, and availability of appropriate end-of-treatment data. Heterogeneity was calculated using $x^{2}$ and $\mathrm{I}^{2}$ statistics. A random effects model was applied to heterogeneous study data. For studies for which a meaningful meta-analysis was precluded, it was decided that data would be qualitatively synthesised.

Of the 106 articles located after the removal of duplicates, 14 articles reporting on randomised, controlled trials (RCTs) met the selection criteria ( $\boldsymbol{O}$ Fig. $\mathbf{1}$ ). However, two articles were based on the same data $[28,29]$. In this case, both papers were examined to extract all relevant information.

\section{Results}

\section{$\nabla$}

\section{Description of studies}

Overall, twelve of the thirteen separate trials are included in the present review. The excluded trial by Gerard House reported results for the active treatment group but failed to include data for the placebo arm, or between-group significance values [30]. It would appear that one report [31] is a subpopulation analysis of a larger multicentre trial [28]. It is unclear whether another of the trials was blinded [32]. Three of the studies were of 2 cycles/ months duration, one continued for 6 cycles; and nine studies lasted 3 cycles/months, one of which, after a 2-month washout period, readministered the same extract only on the last seven days of the luteal phase for a further 3-month period.

Conditions investigated: The characteristics of the identified studies are included in $\odot$ Table 1. Of the 12 RCTs, eight investigated the effects of Vitex extracts in women suffering from PMS, two examined Vitex in PMDD, and the remaining two investigated latent hyperprolactinaemia (LHP) with/without mastalgia.

Controls: Six of the eight PMS studies were placebo-controlled while two were comparator studies, comparing the effects of $\mathrm{Vi}$ tex with pyridoxine (vitamin B6) and magnesium, respectively. Both RCTs on PMDD compared Vitex extracts with fluoxetine. Of 


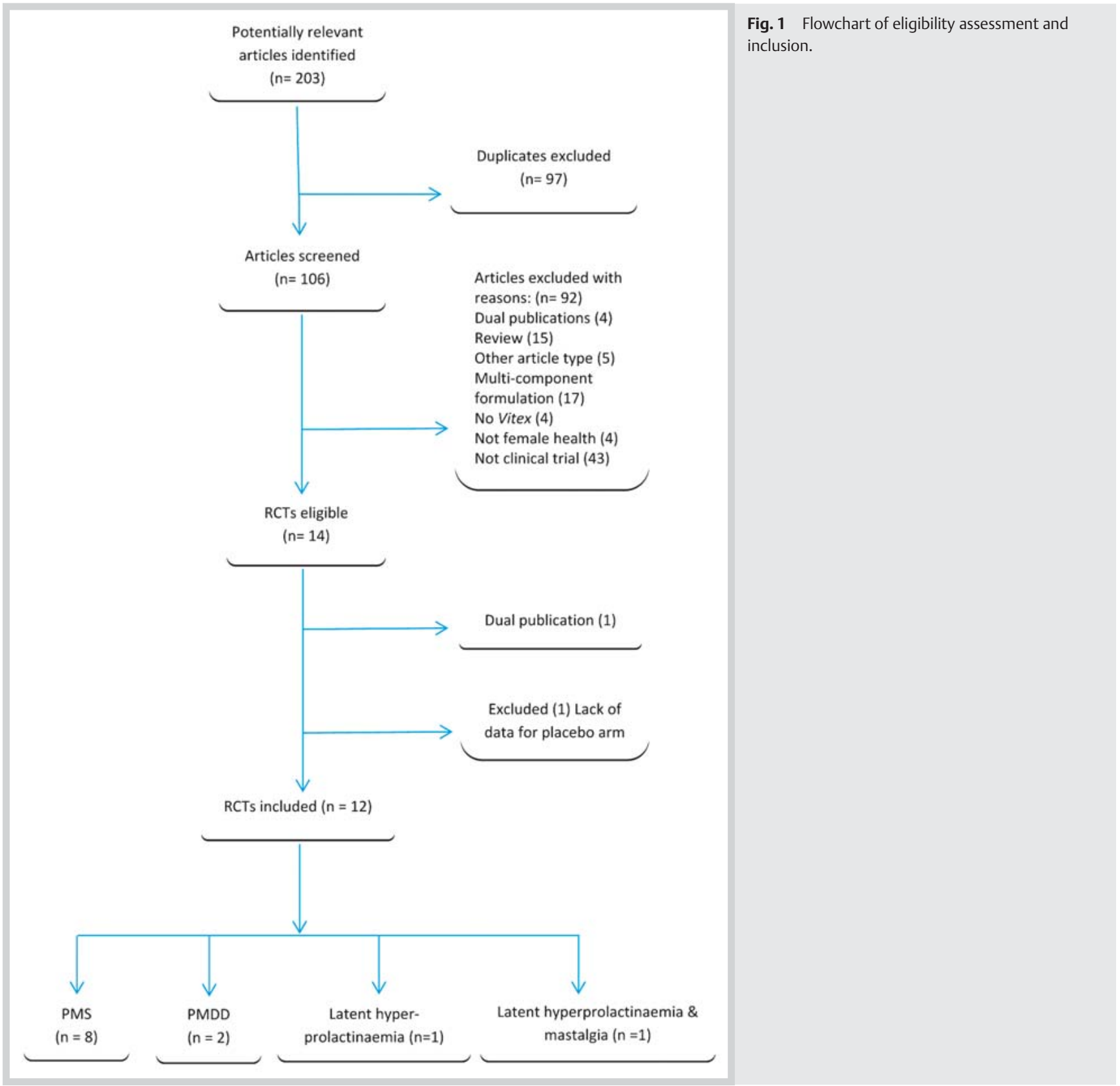

the two trials investigating LHP, one was placebo-controlled, while the other compared Vitex with bromocriptine in LHP or mastalgia.

Participants and settings: In the five studies reporting sample size calculations [23,24,31,33,34], estimates ranged from 55 to 120 patients per arm, allowing for drop-outs and withdrawals. All but one of these [33] retained adequate numbers for analysis. Six of the twelve studies gathered trial data from over 100 participants (range 110 to 217). No differences in results were observed according to setting, which included clinical, university, and community-based levels, with the only negative finding from a community-based trial in which PMS sufferers self-identified.

Baseline symptoms were diagnosed according to the DSM-III or DSM-IV criteria in five PMS studies [22,23, 28,31,35] and for both PMDD studies $[21,36]$. PMS was diagnosed by general practitioners in one other study [34] and was self-diagnosed (1) [24] or selfrated on the PMTS (1) [33] for the remaining PMS trials. LHP was established by the mid-follicular phase prolactin response to TRH stimulation, and serum prolactin levels on days 5-8 of the menstrual cycle, respectively, in the two trials using this endpoint. It was specified in five (of eight) PMS and both PMDD studies that participants experienced regular cycles, with a cycle length ranging between 22 and 35 days (25-34 [23,36]; 24-35 [34]; 22-35 $[28,31])$. Symptoms were required to have been present for the previous three [35], six [36], or 12 months [28]. Five studies reported a requirement for an exacerbation of symptoms in the late luteal phase as compared to the follicular phase (in days 5-10) [23, 36], (days 3-9) [28], (days unspecified) [31,33]. However, only four specified the magnitude of the exacerbation: an increase in the luteal phase (seven days before menses) of at least 16 points on the PMSD scale compared with the follicular phase $[28,31]$ or an increase of $30 \%$ or over (days 23-28) compared with the postmenstrual scores (days 5-10) [23,36]. 


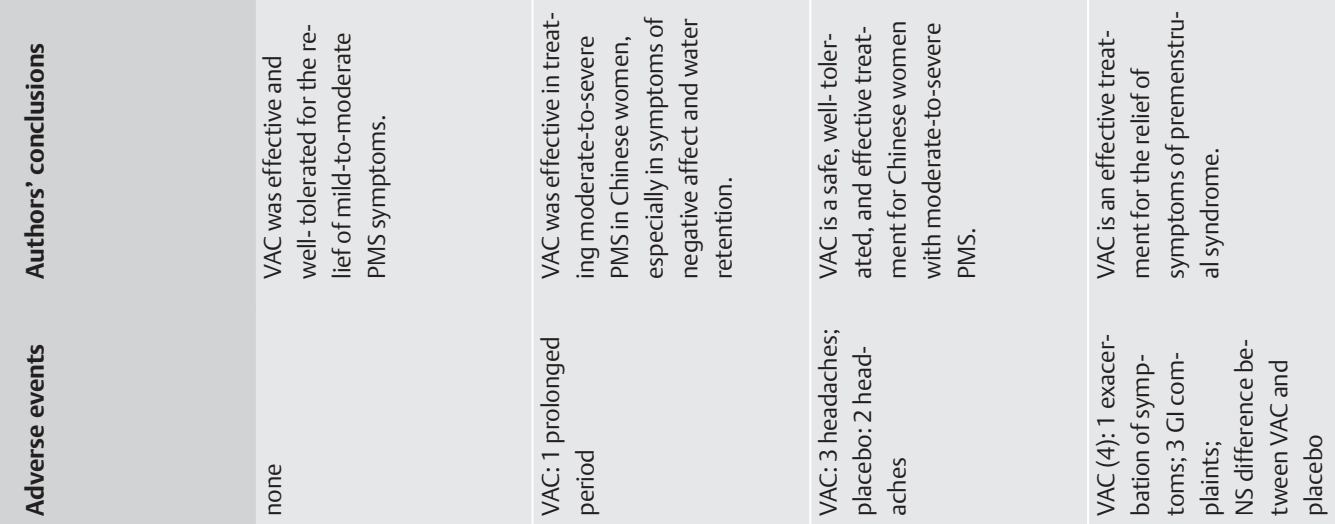

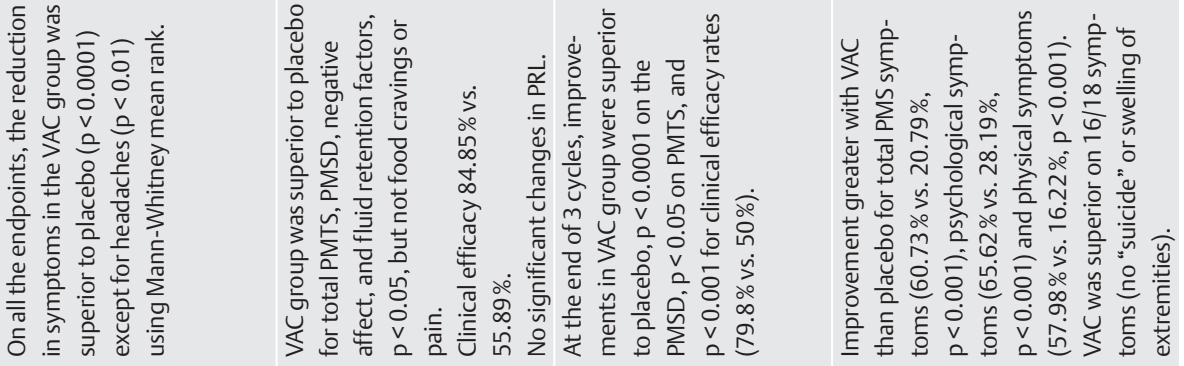

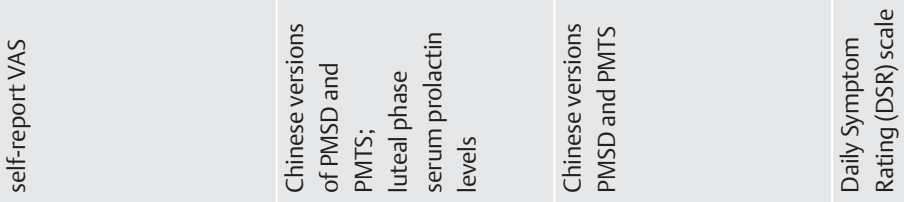

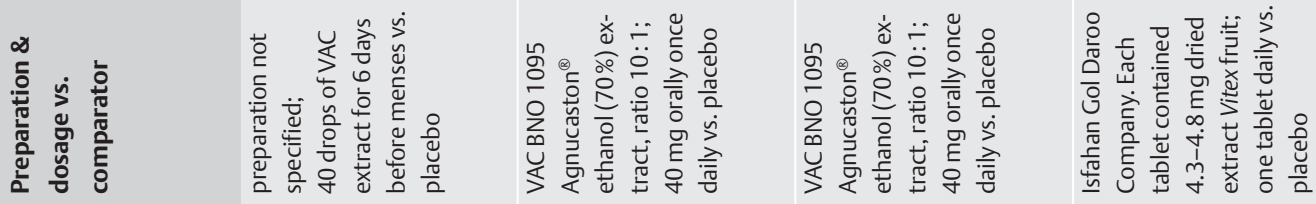
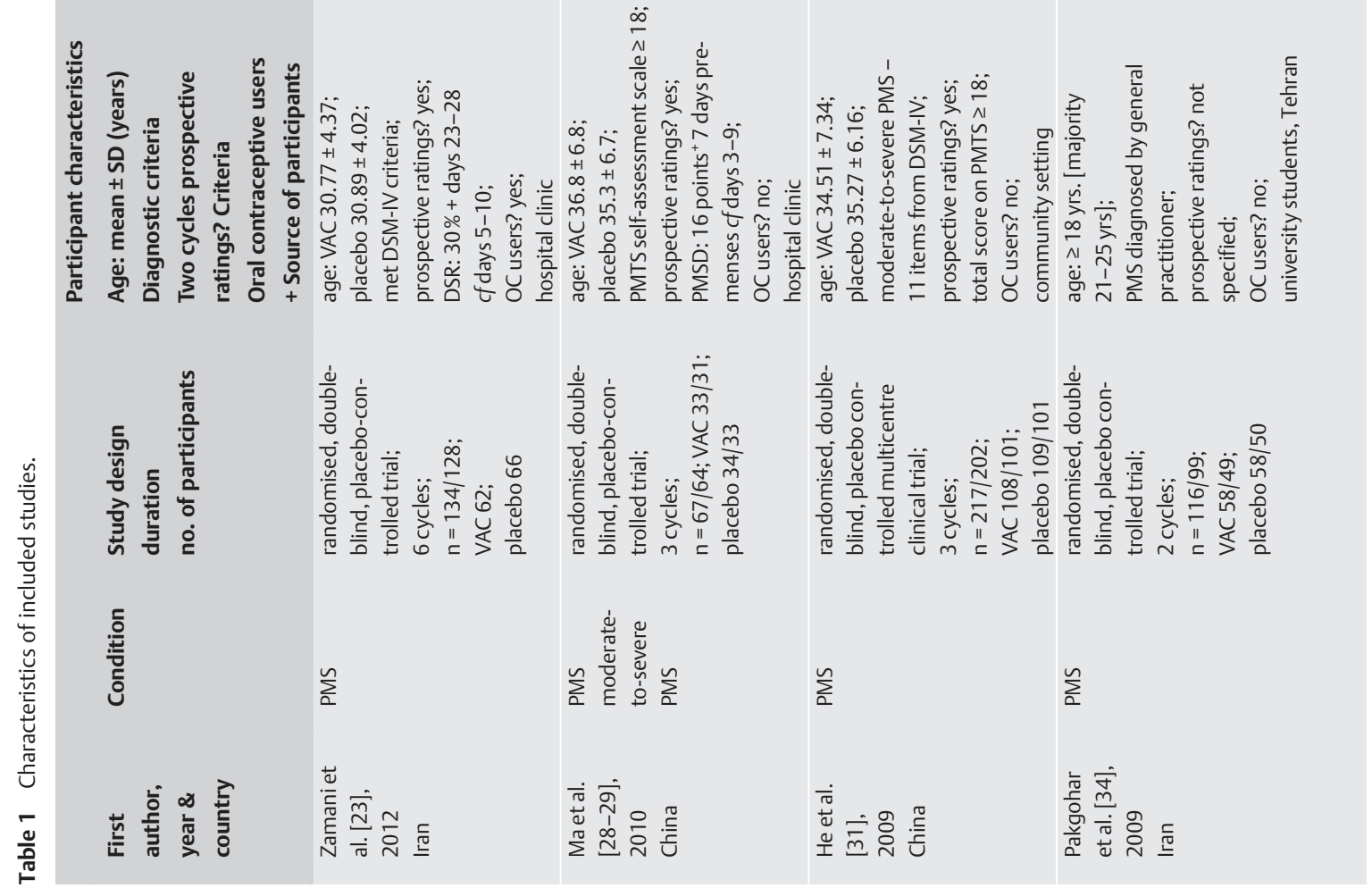


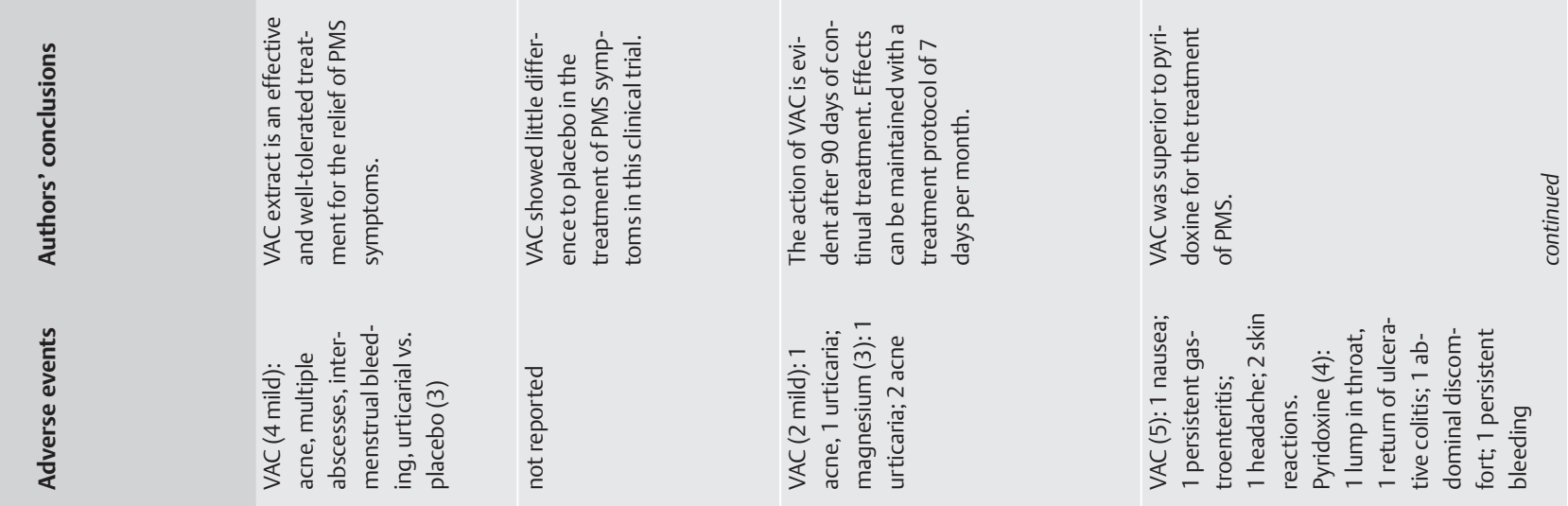

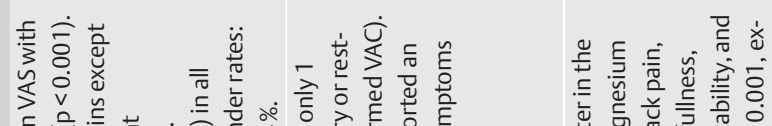

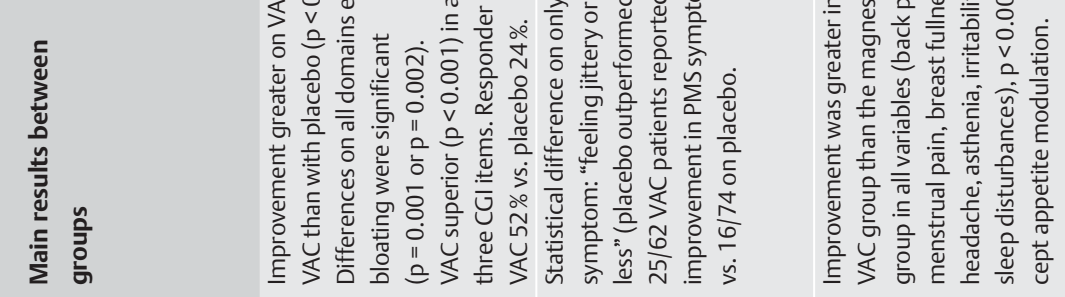

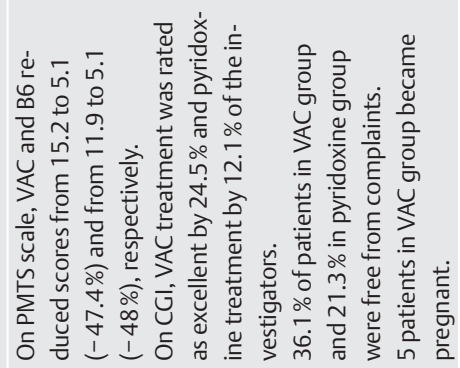

II

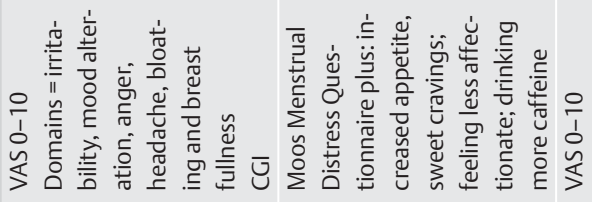

率可

\begin{tabular}{|c|c|c|c|}
\hline 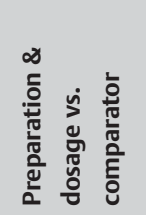 & 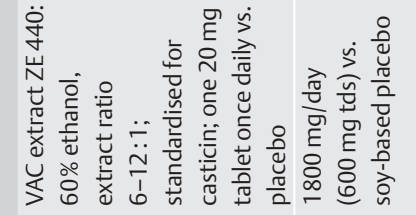 & 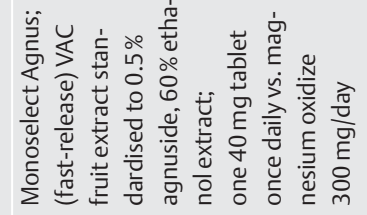 & 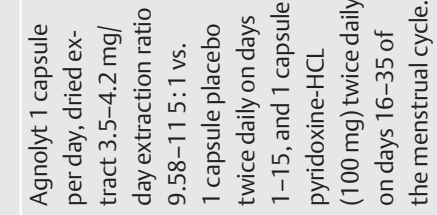 \\
\hline
\end{tabular}

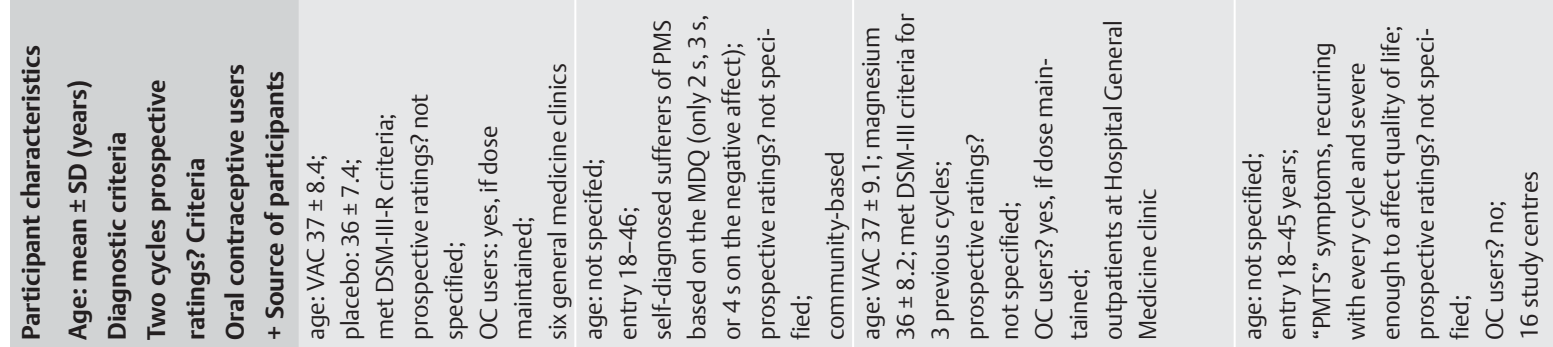

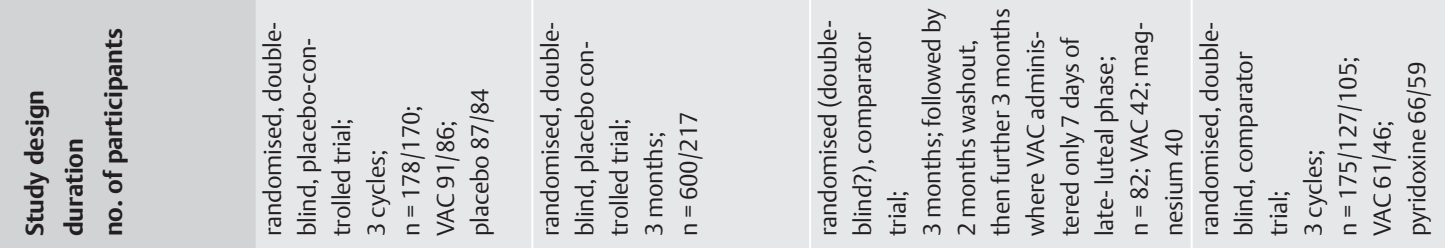

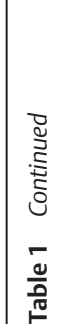
흘
$\sum_{a}^{n}$
$\sum_{\Sigma}^{n}$
$\sum_{\Sigma}^{n}$
$\sum_{\Sigma}^{n}$

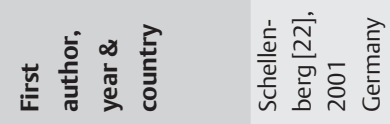
望部

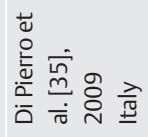

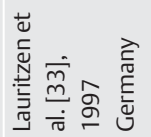



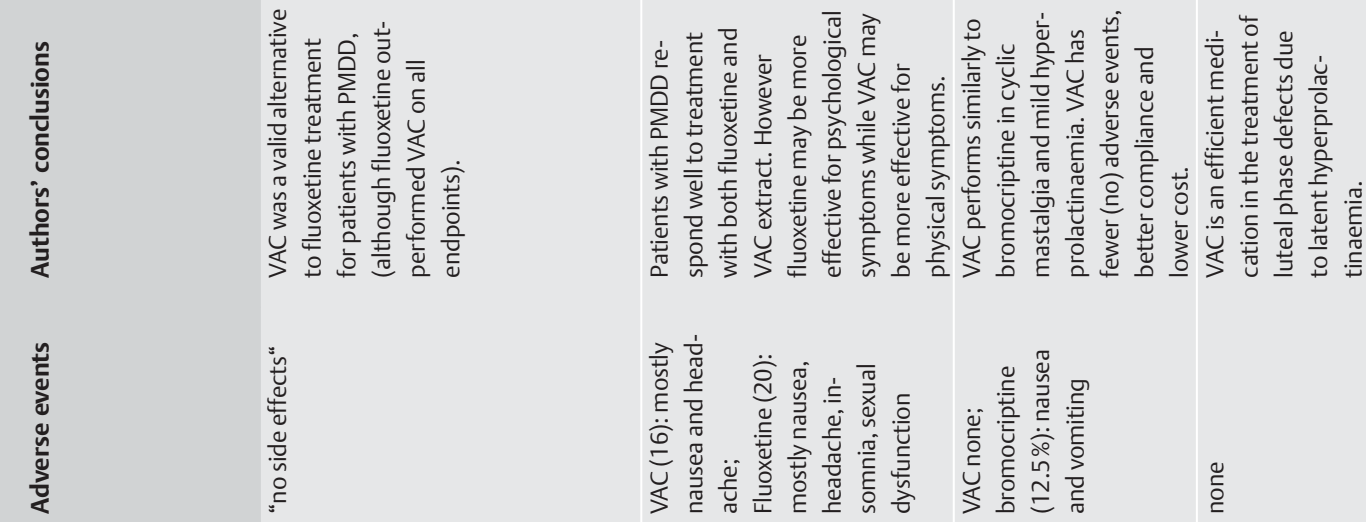

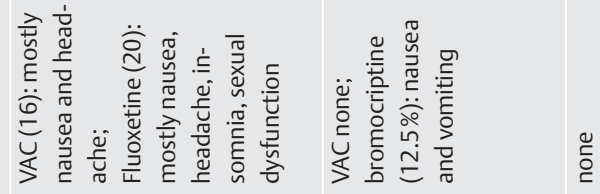

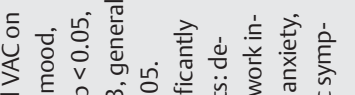

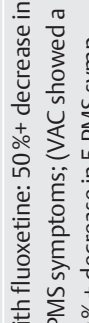

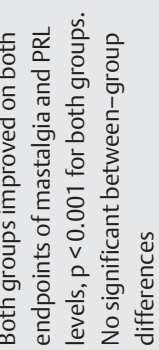

它

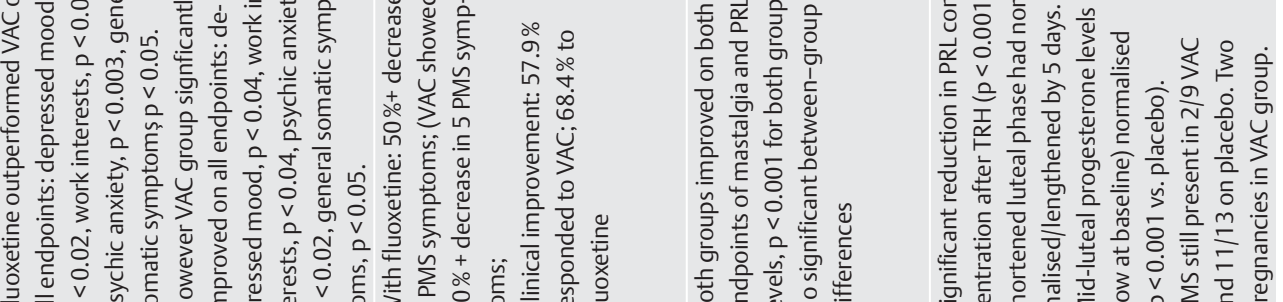

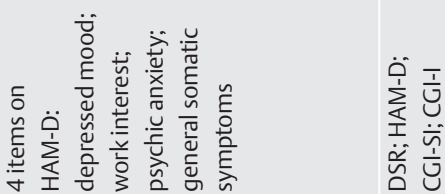

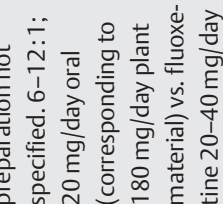

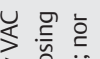

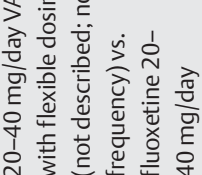

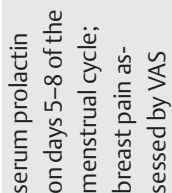

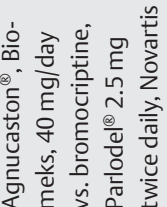

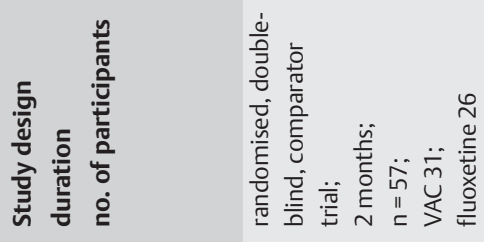

产

|

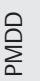

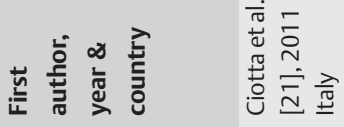

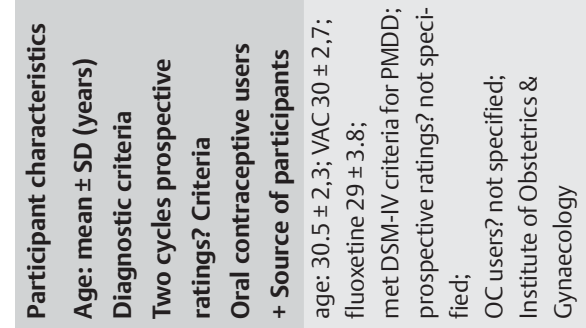

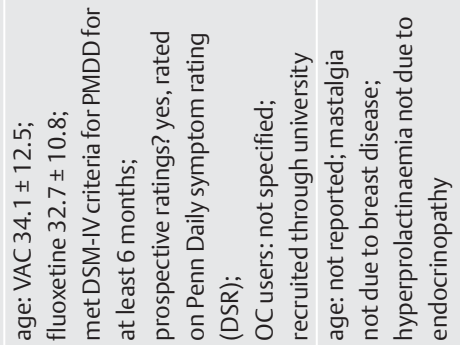

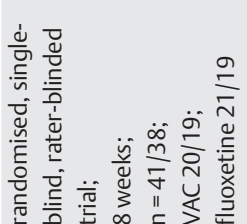

$\sum_{\mathrm{\Sigma}}^{\mathrm{Q}}$

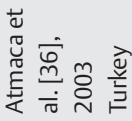

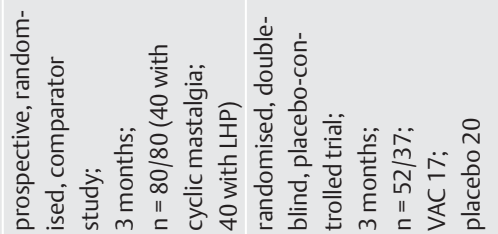

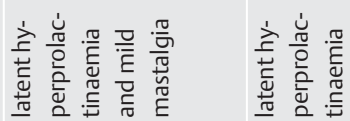

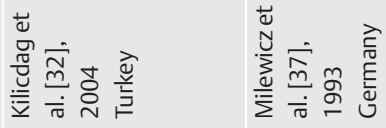


Four studies (of the 10 PMS and PMDD studies for which it was relevant) reported two or more cycles of prospective daily rating of symptoms prior to randomisation. Oral contraceptive (OCP) users were excluded from four of the eight PMS studies $[28,31$, $33,34]$, while three permitted its use providing the dose was maintained $[22,23,35]$. However, outcomes were similar in studies including or excluding OCP use.

In most studies, women in the early perimenopausal age group were eligible for inclusion. Women were accepted up to age 46 (1 study) [24], 45 (3 studies) [31,33,36], 44 (1 study) [28], and 42 ( 1 study) [37]. In a further three [22,34,35], women 18 years and over were recruited, and women of "child bearing age" in another [23]. Age range was not reported in two studies [21,32]. The lower included age varied from 18 to 24 years. There were no reports of the actual age ranges recruited, but means reported ranged from 29 to 37 years.

Interventions: Six different Vitex preparations were specified in eight of the 12 trials, with the remaining four not specifying the product used $[21,23,24,36]$. VAC BNO 1095 Agnucaston ${ }^{\circledR}$ dried ethanolic (70\%) extract/tincture was used in two PMS studies $[28,31]$ as well as in the study on mastalgia and LHP [32]. Of the other studies, each of the following was used in one trial: VAC extract ZE 440: 60\% ethanol m/m, extract ratio 6-12:1, standardised for casticin (PMS trial) [22]; Isfahan Gol Daroo Company containing 4.3-4.8 mg dried extract of Vitex fruit per tablet (PMS) [34]; Agnolyt, 1 capsule per day, dried extract 3.5-4.2 mg/day, extraction ratio 9.58-11.5: 1 (PMS) [33]; Monoselect Agnus, a fastrelease VAC extract standardised for $0.5 \%$ in agnuside (PMS) [35]; and Strotan capsules, aqueous ethanolic 50-70\% extract of VAC dried fruits (LHP) [37].

Dosages and dosing regimens varied from 40 drops (approximately $2 \mathrm{~mL}$ ) of VAC extract/tincture for 6 days before menses [23] to $1800 \mathrm{mg} /$ day [24]. The most commonly prescribed dose was $40 \mathrm{mg} /$ day dried fruit equivalent (DFE) [28,31-33], although $20 \mathrm{mg} /$ day was administered in one trial [37] and a flexible dose of $20-40 \mathrm{mg} /$ day (presumably DFE, not extract) in one of the PMDD studies [36]. In the other PMDD study, an extract 6-12:1, $20 \mathrm{mg} /$ day oral (corresponding to $180 \mathrm{mg} /$ dry plant material) was administered [21]. In one key PMS trial, the daily dose was $180 \mathrm{mg} /$ day DFE [22]. For one other trial, it was difficult to definitively determine the DFE dose, although it was probably $40 \mathrm{mg} /$ day [34]. Treatment was generally administered once daily for the entire month.

Comparators: In the RCTS on PMS, placebo tablets described in one trial were soy-based [24]. Other comparators were one capsule placebo twice daily on days 1-15 and one capsule pyridoxine-HCL (100 mg) twice daily on days 16-35 of the menstrual cycle [33], as well as $300 \mathrm{mg}$ magnesium oxide daily [35]. Both PMDD studies compared Vitex extracts with fluoxetine 20$40 \mathrm{mg} /$ day $[21,36]$. In the trial investigating LHP and mastalgia, the comaparator was bromocriptine, Parlodel ${ }^{\circledR} 2.5 \mathrm{mg}$ twice daily, Novartis [32].

\section{Outcome measures}

Eight different outcome measures were used in the RCTs investigating PMS and PMDD. Among the PMS studies, two measured symptoms on the premenstrual tension syndrome self-rating scale (PMTS) and premenstrual syndrome diary (PMSD) but could not be combined into a meta-analysis due to apparently not being independent. Although two others used visual analogue scales, these could not be combined for analysis as one reported total symptoms (mean difference) while the other re- ported the means of individual symptom improvements [without standard deviations (SDs)]. Further analysis was precluded as different measures were employed by all the other PMS studies, and not all reported end-of-treatment means and SDs. Four authors were contacted for these details [21-24]; two provided data, where normal distribution permitted [22,23]. Similarly, the HAM-D was used as an outcome measure for both PMDD studies, but one reported on only four domains, while the other reported total scores. No meaningful analysis could be undertaken for the LHP endpoint as different controls were used. None of the studies reported effect sizes or confidence intervals.

\section{Trial results}

Results of identified studies are summarised in Tables $\mathbf{1}$ and $\mathbf{2}$. Despite the heterogeneity of the studies and outcome measures, the results were generally consistent. In the treatment of both physical and psychological PMS symptoms, Vitex extracts were shown to be superior to placebo in all but one of the six studies described as placebo-controlled. Due to heterogeneity of the disorders, comparators, endpoints, limited availability of appropriate end-of-treatment data, and two studies not appearing to be independent, it was only possible to perform a meta-analysis on two PMS studies. Combining the results of the multicentre study reporting total PMS symptoms on the PMSD and PMTS scales [31] with the outcome reported on the Penn's DSR [34], Vitex also showed a greater benefit than placebo (MD 8.38, 95\% CI 3.48 to 13.28 for PMSD; MD 7.71, 95\% CI 1.24 to 14.17 for PMTS) (๑ Fig. 2).

\section{PMS: Vitex versus placebo}

In the four placebo-controlled trials reporting significance values for total PMS scores, Vitex extracts were found to be superior to placebo. In a multicentre trial with 208 participants, VAC BN01095 (40 mg/day, 70\% extract Agnucaston ${ }^{\circledR}$ ) was superior to placebo over 3 cycles for total PMS symptoms measured on the PMTS ( $p<0.001)$ and PMSD ( $p<0.05)$ scales, and clinical efficacy rates $(p<0.001)$ [31]. The same results were observed in what appears to be a subset of these women at a hospital in Beijing for total PMS symptoms on PMTS and PMSD, and for negative affect and water retention (all $\mathrm{p}<0.05$ ) but not pain or food cravings [28]. However, on the individual symptoms, 16 of 17 (all except pain) showed superiority over placebo $(p<0.001)$ in this same cohort [29]. Improvement was greater with the Vitex extract (4.3-4.8 mg/day dried extract, Isfahan Gol Daroo) than with placebo for total PMS symptoms measured on the DSR over 2 cycles, and for psychological and physical domains (both $\mathrm{p}<0.001$ ) but not symptoms of "suicide" or swelling of extremities [34]. Over three cycles, Vitex extract ZE 440 (20 mg/day dried extract; ratio $6-12: 1,60 \%$ ethanol) was found to be superior to placebo in 170 women with mild-to-moderate PMS symptoms, for total PMS symptoms measured on a VAS and CGI $(\mathrm{p}<0.001)$ and for all domains [irritability, mood alteration, anger, and breast fullness ( $\mathrm{p}<0.001)$; as well as headache $(\mathrm{p}=0.002)]$ except bloating [22].

On individual symptoms/symptom-clusters, four of five studies reported superiority of Vitex extracts over placebo. In addition to those cited above, symptom improvement was noted in 128 women with 40 drops of Vitex/day (extract not specified) over 6 cycles in symptoms measured on a VAS: nervousness, restlessness, depression, breast pain, bloating (all p <0.001), as well as headaches, $(p<0.05)$ [23]. One (weak) study found that the soybased "placebo" used significantly outperformed Vitex, $1800 \mathrm{mg} /$ 


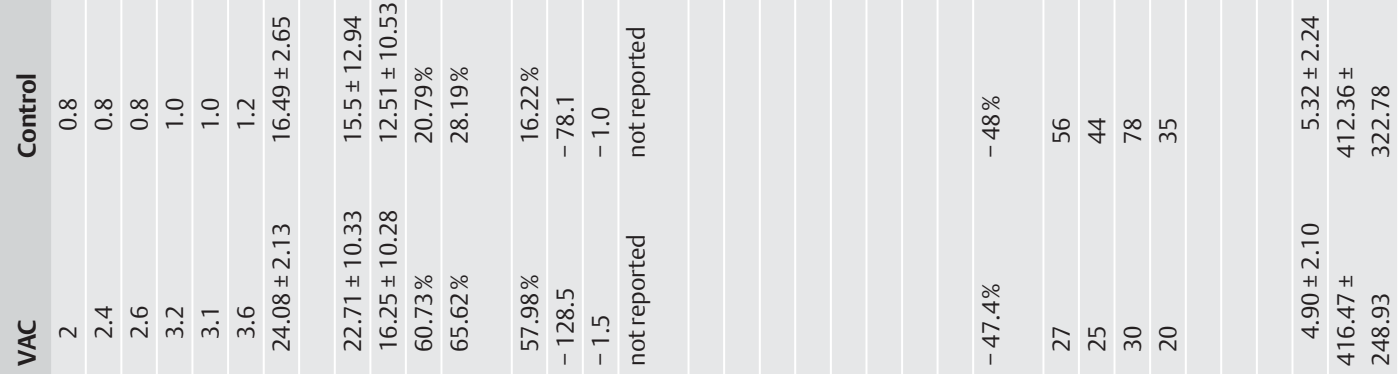

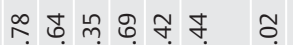

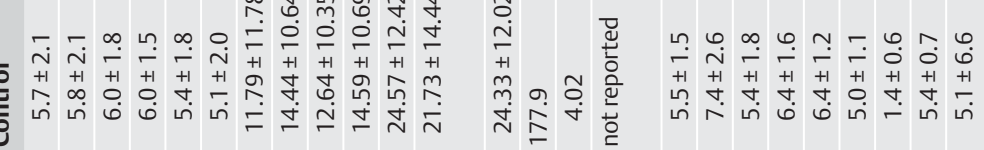

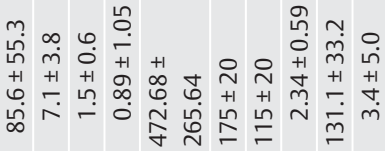
蓄

ㄴำ

웅요

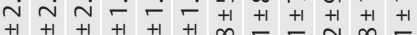
荡

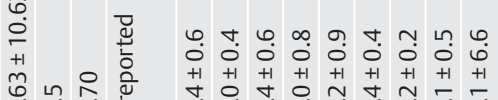

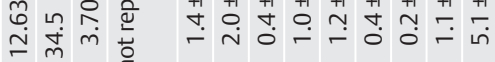

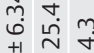

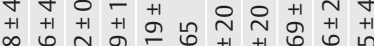

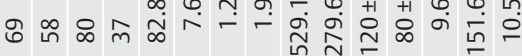

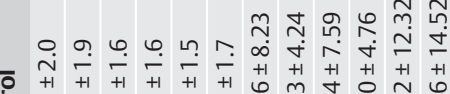

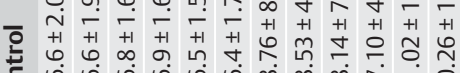

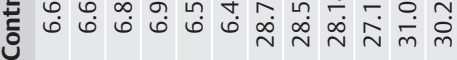

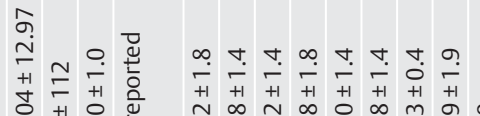
तi 品 in

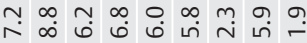
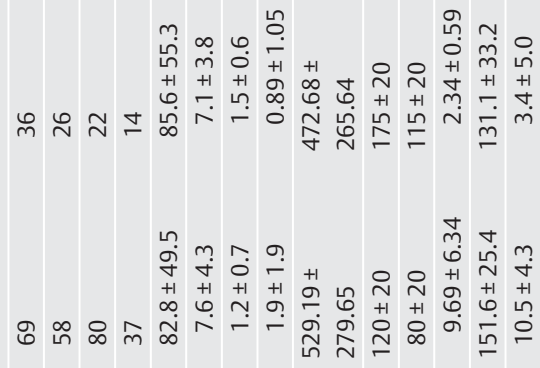

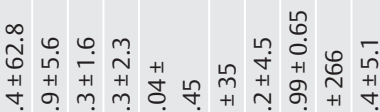
๙

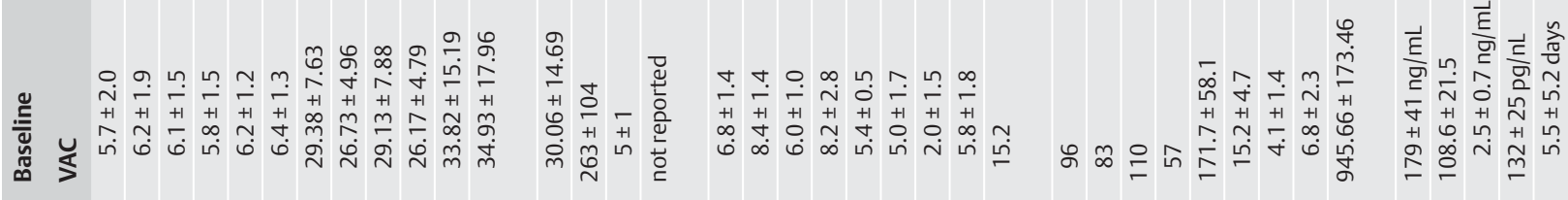

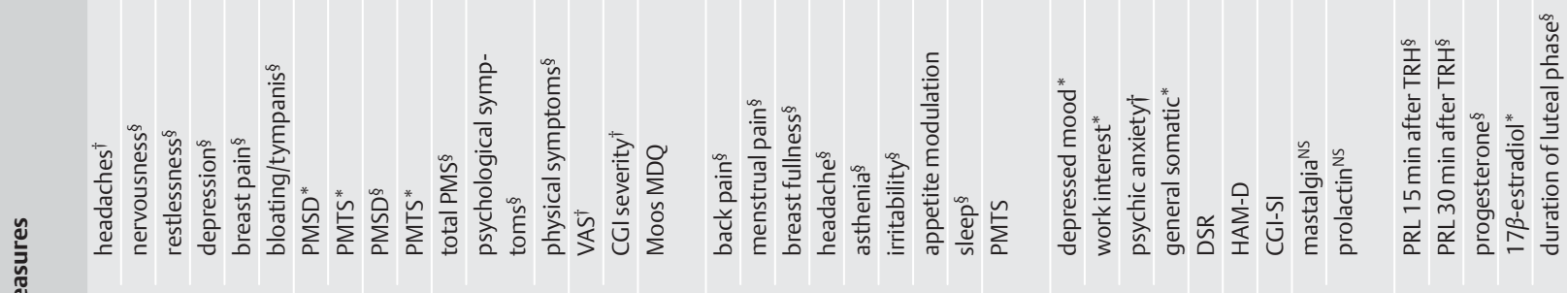

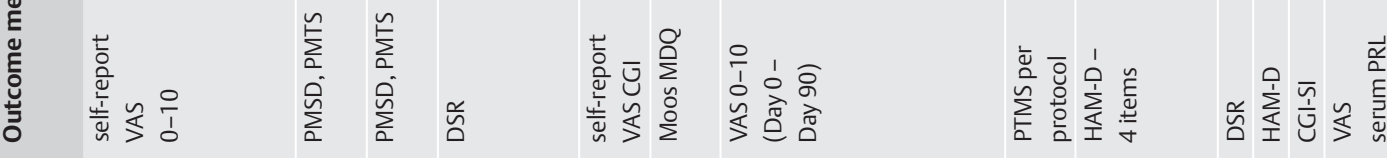

$+$<smiles>C1CCCC1</smiles>

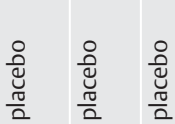

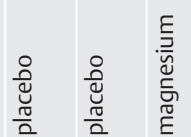

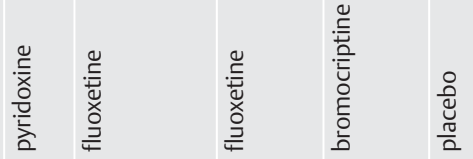

㐫 $\sum_{a} \sum_{\Sigma}^{n} \sum_{\Sigma}^{n} \sum_{a}^{n} \sum_{a}^{n}$

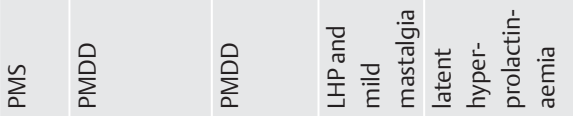

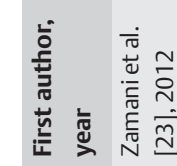

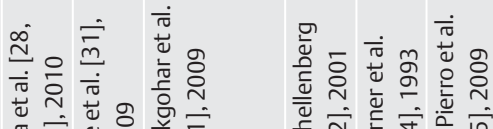

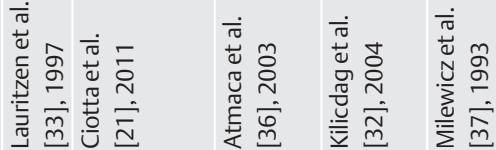




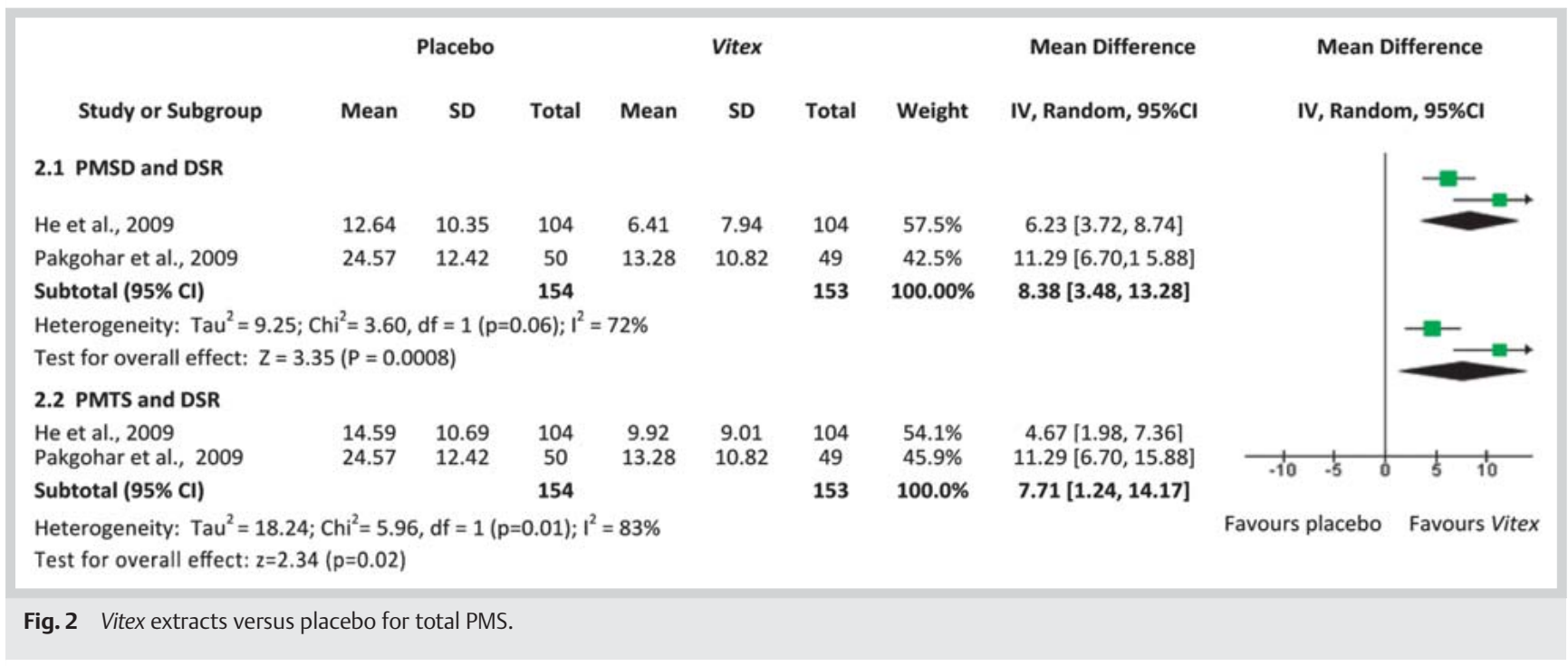

day (extract not specified), on the symptom of feeling jittery/ restless $(\mathrm{p}<0.05)[24]$.

\section{PMS: Vitex versus other comparator}

Two studies compared Vitex extracts with other comparators for PMS. In 127 women over three cycles, Vitex (3.5-4.2 mg/day Agnolyt ${ }^{\circledR}$, extraction ratio $\left.9.58-11 ; 5: 1\right)$ performed similarly to pyridoxine (vitamin B6, $200 \mathrm{mg} /$ day) for PMS, with overall reductions in total symptoms measured on the PMTS of $47.4 \%$ and $48 \%$, respectively; pregnancy occurred in 5 patients in the Vitex group [33]. An RCT involving 124 women found that in comparison with magnesium oxide (300 mg/day), Vitex ( $40 \mathrm{mg} /$ day, MonoselectAgnus ${ }^{\circledR}, 60 \%$ ethanol extract) over 3 months showed superiority on back pain, menstrual pain, breast fullness, headaches, asthenia, irritability, and sleep (all p < 0.001), but not appetite modulation [35]. After a 2-month washout period when symptoms returned to baselines scores, Vitex administered to 21 women for a further 3 months, only in the last 7 days of the luteal phase, was superior to no treatment $(n=21)$ on all the same endpoints $(\mathrm{p}<0.01)$.

\section{PMDD: Vitex versus fluoxetine}

Two studies compared Vitex extracts with fluoxetine in PMDD. Clinical improvements were observed over 8 weeks in $57.9 \%$ of 20 participants administered Vitex (20-40 mg/day, extract not specified) compared with $68.4 \%$ of the 21 who received fluoxetine $(20-40 \mathrm{mg} /$ day), with a $50 \%$ or greater response on 5 domains with Vitex (irritability, breast tenderness, swelling, food cravings, and cramps) compared with 7 with fluoxetine (depression, irritability, insomnia, nervous tension, feeling out of control, breast tenderness, and aches). It was concluded that fluoxetine may be more effective for psychological symptoms, while Vitex may be more effective for physical symptoms [36]. The second study, involving 57 women for 2 months, found that, despite significant improvements from baseline in both arms for all outcomes, fluoxetine (20-40 mg/day) outperformed Vitex (40 mg/ day, 6-12:1, extract not specified) on all endpoints: depressed $\operatorname{mood}(\mathrm{p}<0.02)$, work interests $(\mathrm{p}<0.05)$, psychic anxiety $(\mathrm{p}<0.003)$, and general somatic symptoms $(\mathrm{p}<0.05)[21]$.

\section{Latent hyperprolactinaemia}

Two studies investigated Vitex extracts in latent hyperprolactinaemia. In one of these, a placebo-controlled trial involving 52 women, Strotan ${ }^{\circledR}$ capsules ( $20 \mathrm{mg} /$ day DFE) were administered in the evening on an empty stomach for 3 months [37]. Compared with placebo, prolactin concentration after TRH stimulation was signifcantly reduced $(\mathrm{p}<0.001)$, luteal phase was normalised (lengthened by five days, $\mathrm{p}<0.001$ ), mid-luteal progesterone levels were normalised $(\mathrm{p}<0.001)$, and $\beta$-oestradiol was significantly increased $(\mathrm{p}<0.05)$. In the other study, a prospective, randomised, comparator study of 3 months' duration, the effect of Vitex (40 mg/day DFE Agnucaston ${ }^{\circledR}$, Biomeks) was compared with bromocriptine ( $5 \mathrm{mg} /$ day Parlodel ${ }^{\circledR}$, Novartis) in 40 women with mild hyperprolactinaemia and 40 with cyclic mastalgia [32]. A signficant within-group drop in prolactin levels was observed with both treatments ( $\mathrm{p}<0.0001$ for each), with no significant between-groups difference. Similarly, mastalgia assessed on a VAS also decreased significantly in both groups ( $p<0.0001$ for each), with no significant differences observed between groups. Two women in the Vitex arm became pregnant.

\section{Methodological quality}

Assessment of risk of bias: Risk of bias, assessed according to the Cochrane risk of bias criteria, was predominantly low or unclear ( Figs. 3 and 4). High risk of bias was only detected in two of the studies [32,33], on one, two, and three of the criteria, respectively ( Fig. 3). Overall, low risk of bias was most commonly identified for reporting bias (all studies), selection [22,24,28,31,33$35,37]$ and attrition bias [22,28,31,32,34-37] (eight studies each). Blinding of participants and personnel (performance bias) was most commonly inadequately described (unclear in 10 studies [21-24,28,31,35-37], and high risk in one [32]); followed by random sequence generation (unclear in eight studies [21,24, 28, 31-35]) and blinding of outcome assessment (unclear in six studies $[21,24,28,31,35,36])$. It would appear that one of the studies with high risk of bias on two criteria was not blinded as both interventions were propriety products, and a double-dummy design was not described [32].

Assessment on Jadad scale: On the Jadad scale, of the twelve identified randomised, controlled trials, eight were rated with a score 


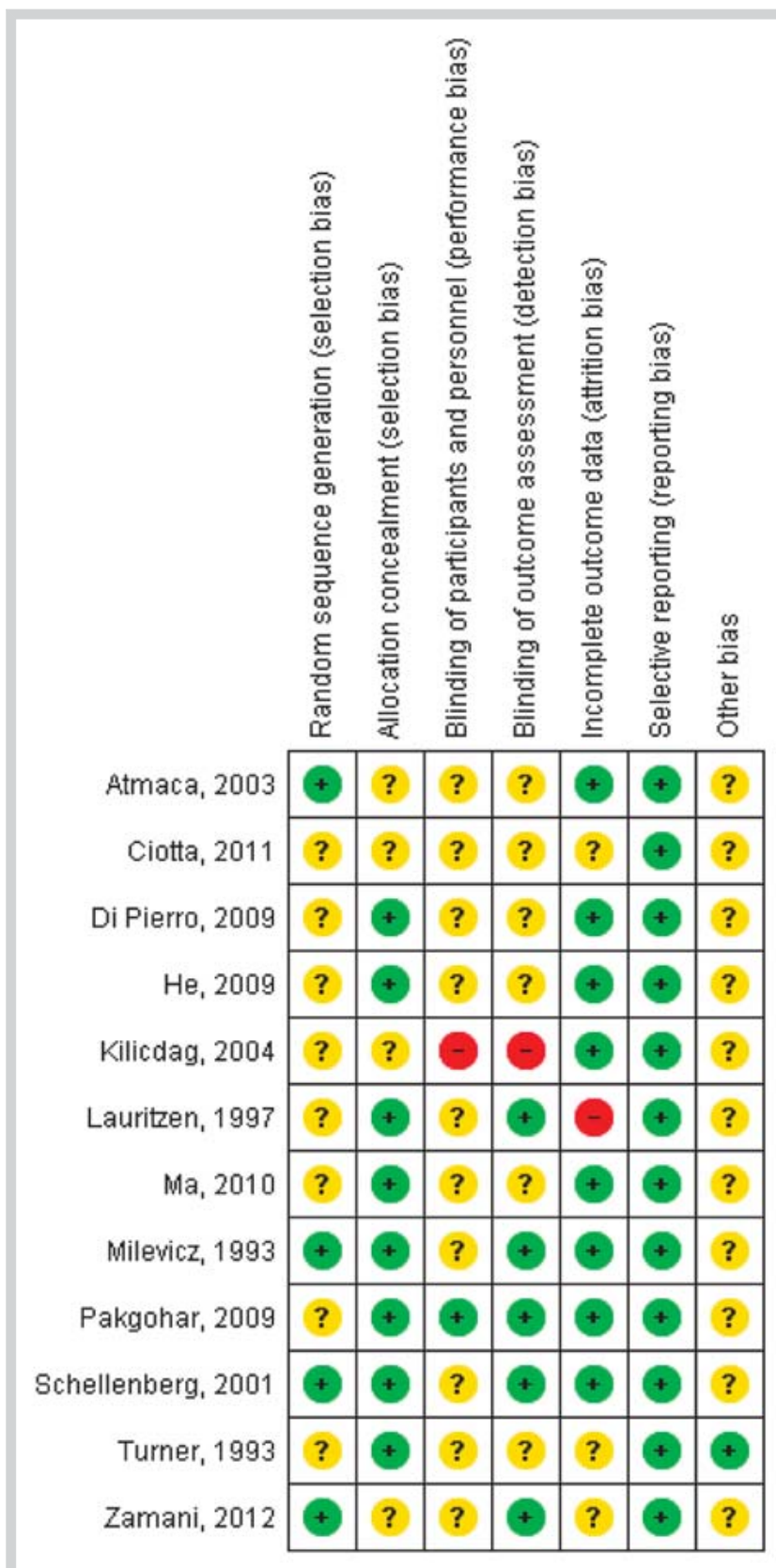

Fig. 3 Risk of bias: summary. of $4[22,23,28,31,33,34,36,37]$, and four with a score of 2 [21, $24,32,35]$.

Assessment of reporting according to elaborated CONSORT statement for trials of herbal interventions: All identified studies were assessed according to the elaborated CONSORT statement for trials of herbal interventions [27] ( Table 3). Information was reported in all studies on the Latin binomial, dose and duration of administration; the plant part was reported in all but one study. Eight studies included information on the propriety product name or extract name and name of manufacturer of the product (4A.2); seven studies specified the type of extract. All other items were infrequently reported. Details relating to qualitative testing and practitioners were rarely provided, and no studies provided information about authorisation (licensing or registration of the product [4A.3]), authentication of raw material, and retention of a specimen (4B.4), or content constituents per dosage unit form, and extraneous materials (4C.2). No study reported more than 10 (of a possible 24) pieces of information.

Sample size calculations were reported in five studies $[23,24,31$, $33,34]$. Intention-to-treat (ITT) analysis was specifically reported in three studies $[24,28,31]$. Results were ostensibly based on ITT analyses in a further three, reporting no dropouts or withdrawals $[21,32,35]$. Six other studies described dropouts and withdrawals and provided a per protocol analysis.

\section{Adverse events}

Four studies reported no adverse events in the Vitex arm [21,23, 32,37 , while another failed to report on adverse events [24]. In the other seven trials, adverse events associated with Vitex were found to be mild and not significantly more frequent than with placebo, magnesium, or pyridoxine. Compared with fluoxetine [36] or bromocriptine [32], adverse events with Vitex extracts were reported to be less severe.

In total, 35 adverse events were reported from 641 participants. The highest percentage was reported in a comparator study with fluoxetine in PMDD, where 16 of 38 participants reported mostly nausea or headache [36]. Among the other 603 participants, the main symptoms reported were headaches (4) [31,33], skin reactions (2) [33], acne (2) [22,35], urticaria (2) [22,35], and gastrointestinal complaints (3) [34]. There was one report each of nausea [33], multiple abscesses, intermenstrual bleeding [22], persistent gastroenteritis [33], prolonged menstrual period [28], and exacerbation of PMS symptoms [34].

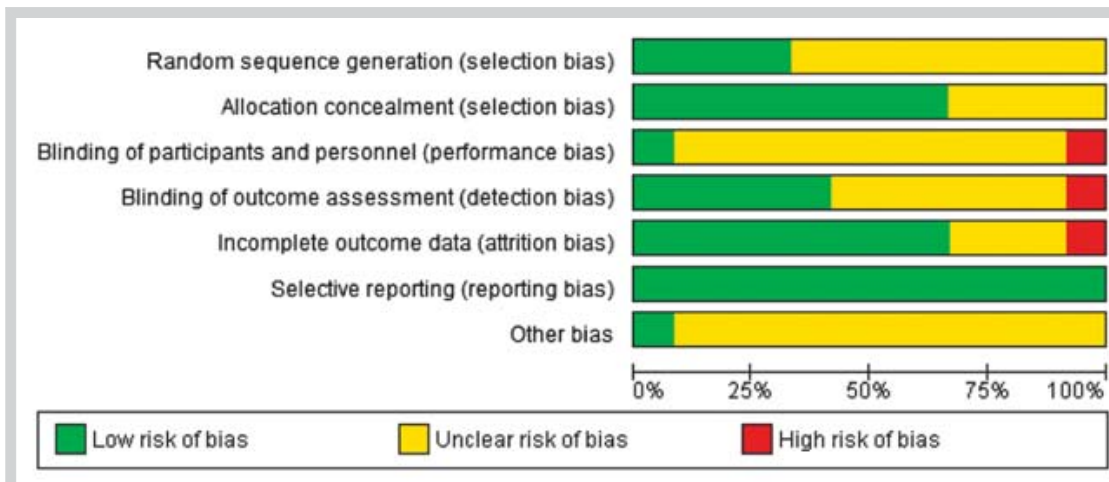

Fig. 4 Risk of bias: graph. 


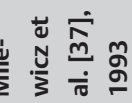

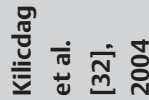

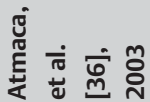

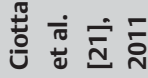

莺 苍 离

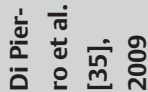

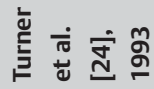

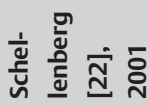

宛 倇

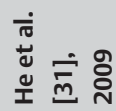

एँ

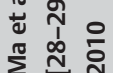

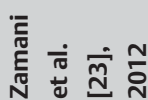

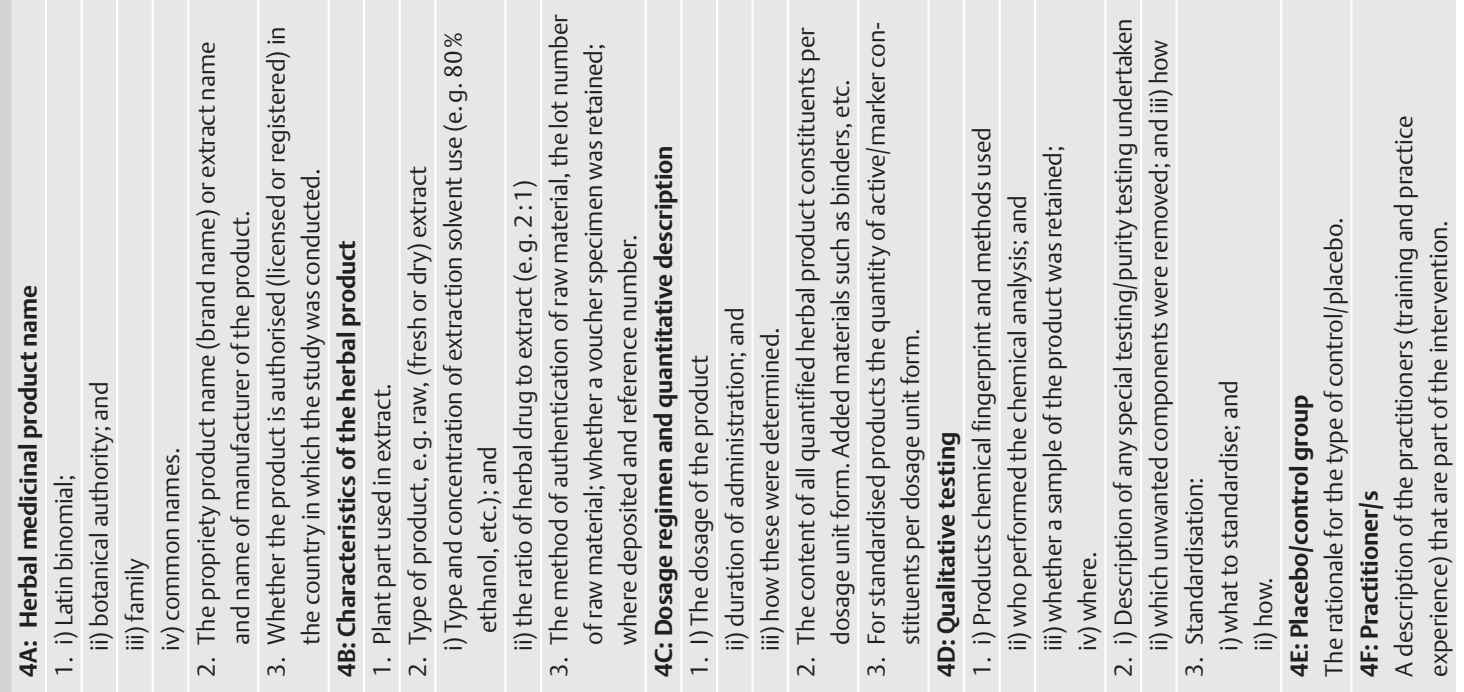




\section{Discussion}

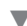

To our knowledge, this is the first systematic review to examine randomised, controlled trials of Vitex agnus-castus extracts in all women's reproductive health conditions. Despite the large number of clinical studies that have been conducted on Vitex in this context, the only randomised, controlled trials that could be identified focused on premenstrual syndrome (PMS) and related conditions, premenstrual dysphoric disorder (PMDD), latent hyperprolactinaemia, and cyclic mastalgia. In all, 106 unique studies were located, and twelve are included in the current review. In PMS, all studies, with the exception of one poor quality trial [24], showed Vitex extracts to be superior to placebo [22,23,28, 29,31,34], pyridoxine (vitamin B6) [33], and magnesium [35] for the amelioration of total PMS symptoms, and psychological and physical subclusters. Findings regarding premenstrual bloating [23], fluid retention [22,28], swelling of extremities [34], and food cravings $[29,36]$ appear to be inconsistent, however. In PMDD, fluoxetine outperformed Vitex in two studies [21,36], although improvements were also evident with Vitex, which was associated with fewer adverse events. For late luteal phase defects due to LHP, one randomised, controlled trial found Vitex to be superior to placebo [37], while a second showed it to be equivalent to bromocriptine but with better compliance and fewer (no) side effects [32]. In the latter study, its effects on cyclic mastalgia were also comparable with those of bromocriptine. The safety profile was excellent overall for Vitex extracts, with adverse events being mild and generally infrequent. The quality of the trials varied from weak to very good, with the majority being rated as good. In terms of risk of bias assessment, three studies were predominantly judged to have a low risk of bias $[22,34$, 37], while the others required greater transparency in reporting. Comparison of studies conducted prior to and post-publication of the CONSORT checklist item 4 for reporting RCTs of herbal medicine suggests that the transparency of reporting has not been impacted by this recommendation.

The findings of the present study are consistent with those of a systematic review of good quality, randomised, controlled trials of phytotherapeutic agents, including Vitex extracts, for premenstrual syndrome [38], and with a review of randomised and nonrandomised studies of Vitex and multicomponent formulations containing Vitex for the treatment of mastalgia [39]. The current systematic review includes a greater number of studies investigating only Vitex mono-preparations, including RCTs of all women's health conditions, and imposing no language restrictions. In addition, we include a risk of bias assessment, assessment of reporting according to the elaboration of the CONSORT checklist item 4 for reporting on RCTs of herbal interventions, and a meta-analysis where studies permitted. The good tolerability and safety profile are consistent with the findings of a 2005 systematic review on the adverse events associated with Vitex extracts [40].

Based on current evidence regarding the pharmacology of Vitex, the following mechanisms may be of relevance: Vitex has been shown to have dopaminergic activity by binding to DA-2 receptors [17], which results in prolactin inhibition. For example, using the corpus striatum membrane dopamine receptor binding assay, it was determined that Vitex contained several active principles that bind to the dopamine D2 receptor. The action of the chaste tree on pituitary hormone secretion in vitro was selective, since both basal and LHRH-stimulated gonadotropin (FSH, LH) release remained unaffected [41]. An extract (containing $3.3 \mathrm{mg} / \mathrm{mL}$ water-soluble substances) markedly reduced stress-induced prolactin release in rats after intravenous injection [15]. However, the prolactin-inhibiting activity is not consistent with a traditional galactagogue action attributed to Vitex, possibly because effects on lactation are dose-related $[41,42]$. Vitex also effected a dose-dependent clinical increase in melatonin secretion [43], suggesting a benefit in the symptoms of disordered sleep associated with female reproductive cycles and in menopause [44,45]. Flavonoids in Vitex (especially apigenin) have also demonstrated binding activity to the beta oestrogen receptor in vitro, a finding of uncertain clinical relevance [46]. The pharmacology of Vitex has been comprehensively reviewed elsewhere $[17,44]$.

The failure to find significance over placebo in the study by Turner et al. could be attributable, at least in part, to their choice of a soy-based placebo. Some evidence suggests that soy may be of benefit in PMS [47], and specifically in physical symptoms of headache, breast tenderness, cramps, and swelling [48]. This is probably due to the mild oestrogen agonist activity of its isoflavone constituents [49]. Therefore, the use of a potentially active placebo was probably ill-advised.

Of the 12 studies reviewed, seven were placebo-controlled while the remaining five were comparator studies. The use of reference treatments that do not have established benefits in premenstrual symptoms such as magnesium or vitamin B6 can potentially cloud the results of these studies. These comparators may have acted as a placebo in these trials. In such instances, the inclusion of a third placebo arm is helpful. By way of elaboration, the interpretation of the finding that Vitex was equivalent to pyridoxine (vitamin B6) on the PMTS scale in the trial by Lauritzen et al. is open to interpretation, since the efficacy of pyridoxine in premenstrual syndrome is controversial $[50,51]$, although there is some evidence to support its role in the treatment of PMS symptoms and premenstrual depression [52,53]. Nonetheless, investigator and patient ratings suggested superiority of Vitex extracts over pyridoxine [33].

The finding that Vitex extracts were better tolerated than bromocriptine in mastalgia patients is of uncertain relevance to current clinical practice as bromocriptine is no longer commonly prescribed, and is known to have a high side effects profile [54, 55].

The diagnosis of latent hyperprolactinaemia based on an abnormally large prolactin response to TRH stimulation is not commonly reported in current literature. However, the effect of Vitex to treat this condition is consistent with its dopaminergic activity.

As is common with research on PMS, consistency was lacking across studies in terms of the definition employed, including degree of aggravation in the late luteal phase, and the specific days of the follicular phase with which these were compared; it was unclear whether some studies required two cycles of prospective daily ratings prior to randomisation. Several studies included perimenopausal women, in whom ovulatory cycles are less frequent, suggesting that some symptoms under examination may have been more appropriately described as PMS-like [56]. The DSM-III and DSM-IV criteria [57] used in several trials for diagnosing premenstrual syndrome describe "late luteal phase dysphoric disorder", and as such these findings appear to relate to PMDD at the more severe end of the PMS spectrum. This suggests a possible lack of uniformity regarding the conditions actually being treated in these studies.

In addition to the limitations mentioned above, publication bias cannot be excluded, particularly in earlier studies published prior 
to the establishment of the international clinical trials register, as most study findings have been positive for Vitex in the context of women's health.

The range of conditions under investigation, outcome measures, and expression of results only allowed for a meta-analysis of two studies to be conducted. The quality of reporting of clinical trials was inadequate to permit a complete assessment of risk of bias. Sample sizes were small in some studies, and sample size calculations were reported in only five. Overall, the wide range of dosages and insufficient information about the Vitex extracts administered prevents determination of phytoequivalence across studies.

Greater transparency in methodological reporting would facilitate assessment of quality of trial design, results, and risk of bias in clinical trials, as would inclusion of full information according to the CONSORT checklist for RCTs of herbal medicine interventions [27]. This latter point would facilitate the determination of phytoequivalence of extracts studied for the purposes of clinical practice as well as further research. To overcome the word limitations, this could be considered as an appendix to the main report [6].

More meaningful interpretation of findings would be permitted by choice of comparators that have clearly established benefits in the condition under investigation and are commonly prescribed in clinical practice.

Despite small sample sizes in some studies, randomised, controlled trials to date appear to support the efficacy and tolerability of Vitex agnus-castus extracts in the treatment of premenstrual syndrome, premenstrual dysphoric disorder, and latent hyperprolactinaemia. However, lack of transparency in the reporting of some studies limits assessment of trial design and, in some cases, results. Future research into Vitex extracts for these conditions would benefit from use of tightly defined patient populations and common endpoints. To permit determination of assessment of risk of bias and phytoequivalence of extracts, greater transparency in reporting methodological details, including the phytotherapeutic intervention, is recommended. This would inform researchers and clinicians prescribing Vitex extracts for premenstrual disorders.

\section{Acknowledgements}

$\nabla$

The authors acknowledge Assoc. Prof. Cliff da Costa, RMIT (statistical input), Vicki Matthews (translation from Italian and administrative assistance), Drs. Maryam Delavari and Behnaz Khosravi (translation from Persian), and Johanna Shergis (Rev Man support).

\section{Conflict of Interest}

$\nabla$

Associate Prof. Kerry Bone is a founder and director of research and development of MediHerb Australia Pty. Ltd. and is related to Diana van Die (in-law).

\section{References}

1 Upchurch DM, Chyu L, Greendale GA, Utts J, Bair YA, Zhang G, Gold EB. Complementary and alternative medicine use among American women: findings from The National Health Interview Survey, 2002. J Women's Health (Larchmt) 2007; 16: 102-113

2 Upchurch DM, Dye CE, Chyu L, Gold EB, Greendale GA. Demographic, behavioral, and health correlates of complementary and alternative medicine and prayer use among midlife women: 2002. J Women's Health (Larchmt) 2010; 19: 23-30

3 Domoney CL, Vashisht A, Studd JW. Use of complementary therapies by women attending a specialist premenstrual syndrome clinic. Gynecol Endocrinol 2003; 17: 13-18

4 Christie S, Walker AF. Vitex agnus-castus L.: (1) A review of its traditional and modern therapeutic use; (2) Current use from a survey of practitioners. Eur J Herbal Med 1997; 3: 29-45

5 [no authors listed]. Agnus castus also effective in fertility disorders. Exeter: The 5th Annual Symposium on Complementary Health Care; 1999

6 Van Die MD, Burger HG, Bone KM, Cohen MM, Teede HJ. Hypericum perforatum with Vitex agnus-castus in menopausal symptoms: a randomized, controlled trial. Menopause 2009; 16: 156-163

7 Singh BB, Berman BM, Simpson RL, Annechild A. Incidence of premenstrual syndrome and remedy usage: a national probability sample study. Altern Ther Health Med 1998; 4: 75-79

8 Winer SA, Rapkin AJ. Premenstrual disorders: prevalence, etiology and impact. J Reprod Med 2006; 51: 339-347

9 Halbreich U, Borenstein J, Pearlstein T, Kahn LS. The prevalence, impairment, impact, and burden of premenstrual dysphoric disorder (PMS/ PMDD). Psychoneuroendocrinology 2003; 28 (Suppl. 3): 1-23

10 Frackiewicz EJ, Shiovitz TM. Evaluation and management of premenstrual syndrome and premenstrual dysphoric disorder. J Am Pharm Assoc (Wash) 2001; 41: 437-447

11 Ader DN, South-Paul J, Adera T, Deuster PA. Cyclical mastalgia: prevalence and associated health and behavioral factors. J Psychosom Obstet Gynaecol 2001; 22: 71-76

12 Halbreich U, Ben-David M, Assael M, Bornstein R. Serum-prolactin in women with premenstrual syndrome. Lancet 1976; 2: 654-656

13 Muhlenstedt D, Bohnet HG, Hanker JP, Schneider HP. Short luteal phase and prolactin. Int J Fertil 1978; 23: 213-218

14 Dietrich M, Hinney B, Link M, Kuhn W, Wuttke W. Latent hyperprolactinaemia as a cause of mastodynia and luteal function impairment. Kyoto, Japan: 5th International Congress Prolactin; 1988

15 Wuttke W, Gorkow C, Jarry H. Dopaminergic compounds in Vitex agnus-castus. In: Leow D, Rietbrock N, editors. Phytopharmaka in Forschung und klinischer Anwendung. Darmstadt: Steinkopff Verlag; 1995: 81-91

16 Yen S. The human menstrual cycle: neuroendocrine regulation. In: Yen S, Jaffe RB, Barbieri RL, editors. Reproductive endocrinology. Physiology, pathophysiology and clinical management. Philadelphia: WB Saunders Company; 1999: 191-217

17 Wuttke W, Jarry H, Christoffel V, Spengler B, Seidlova-Wuttke D. Chaste tree (Vitex agnus-castus)-pharmacology and clinical indications. Phytomedicine 2003; 10: 348-357

18 Wuttke W. The use of chasteberry (Vitex agnus castus) extract in gynecology. Gynäkol Endokrinol 2008; 6: 82-86

19 Noack M. Our experiences with Agnus castus oligoplex for increasing milk yield. Dtsch Med Wochenschr 1943; 9: 204-206

20 Bautze HJ. Investigations on the influence of Agnus castus on the breastfeeding performance. Medwelt 1953; 4: 189-190

21 Ciotta L, Pagano I, Stracquadanio M, Di Leo S, Ando A, Formuso C. Psychic aspects of the premenstrual dysphoric disorders. New therapeutic strategies: our experience with Vitex agnus castus. Minerva Ginecol 2011; 63: 237-245

22 Schellenberg $R$. Treatment for the premenstrual syndrome with agnus castus fruit extract: prospective, randomised, placebo controlled study. BMJ 2001; 322: 134-137

23 Zamani M, Neghab N, Torabian S. Therapeutic effect of Vitex agnus castus in patients with premenstrual syndrome. Acta Med Iran 2012; 50 : 101-106

24 Turner S, Mills SY. A double-blind clinical trial on a herbal remedy for premenstrual syndrome: a case study. Complement Ther Med 1993; 1: 73-77

25 Higgins JPT, Green S. Cochrane handbook for systematic reviews of interventions (version 5.0.1). The Cochrane Collaboration; 2008. Available at http://www.cochrane-handbook.org. Accessed May 13, 2012 
26 Jadad AR, Moore RA, Carroll D, Jenkinson C, Reynolds DJ, Gavaghan DJ, McQuay HJ. Assessing the quality of reports of randomized clinical trials: is blinding necessary? Control Clin Trials 1996; 17: 1-12

27 Gagnier JJ, Boon H, Rochon P, Moher D, Barnes J, Bombardier C. Reporting randomized, controlled trials of herbal interventions: an elaborated CONSORT statement. Ann Intern Med 2006; 144: 364-367

28 Ma L, Lin S, Chen R, Wang X. Treatment of moderate to severe premenstrual syndrome with Vitex agnus castus (BNO 1095) in Chinese women. Gynaecol Endocrinol 2010; 26: 612-616

29 Ma L, Lin S, Chen R, Zhang Y, Chen F, Wang X. Evaluating therapeutic effect in symptoms of moderate-to-severe premenstrual syndrome with Vitex agnus castus (BNO 1095) in Chinese women. Aust N Z J Obstet Gynaecol 2010; 50: 189-193

30 [no authors listed]. Agnus castus. UK: Gerard House Promotional Brochure; 1988

31 He Z, Chen R, Zhou Y, Geng L, Zhang Z, Chen S, Yao Y, Lu J, Lin S. Treatment for premenstrual syndrome with Vitex agnus castus: A prospective, randomized, multi-center placebo controlled study in China. Maturitas 2009; 63: 99-103

32 Kilicdag EB, Tarim E, Bagis T, Erkanli S, Aslan E, Ozsahin K, Kuscu E. Fructus agni casti and bromocriptine for treatment of hyperprolactinemia and mastalgia. Int J Gynaecol Obstet 2004; 85: 292-293

33 Lauritzen C, Reuter HD, Repges R, Bohnert KJ, Schmidt U. Treatment of premenstrual tension syndrome with Vitex agnus castus - Controlled, double-blind study versus pyridoxine. Phytomedicine 1997; 4: 183189

34 Pakgohar M, Moradi M, Jamshidi AH, Mehran A. Assessment of Vitex agnus-castus $L$. extract effect on treatment of premenstrual syndrome. J Med Plants 2009; 8: 98-107, 185

35 Di Pierro F, Prazzoli R, Candidi C, Attolico M. Premenstrual syndrome: Controlled clinical trial with a fast acting form of a highly standardized extract of Vitex agnus castus. Giorn It Ost Ginecol 2009; 31: 153-157

36 Atmaca M, Kumru S, Tezcan E. Fluoxetine versus Vitex agnus castus extract in the treatment of premenstrual dysphoric disorder. Hum Psychopharmacol 2003; 18: 191-195

37 Milewicz A, Gejdel E, Sworen H, Sienkiewicz K, Jedrzejak J, Teucher T, Schmitz H. Vitex agnus castus extract in the treatment of luteal phase defects due to latent hyperprolactinemia. Results of a randomized placebo-controlled double-blind study. Arzneimittelforschung 1993; 43: $752-756$

38 Dante G, Facchinetti F. Herbal treatments for alleviating premenstrual symptoms: a systematic review. J Psychosom Obstet Gynaecol 2011; 32: 42-51

39 Carmichael AR. Can Vitex agnus castus be used for the treatment of mastalgia? What is the current evidence? Evid Based Complement Alternat Med 2008; 5: 247-250

40 Daniele C, Thompson Coon J, Pittler MH, Ernst E. Vitex agnus castus: a systematic review of adverse events. Drug Saf 2005; 28: 319-332
41 Jarry H, Leonhardt S, Gorkow C, Wuttke W. In vitro prolactin but not LH and FSH release is inhibited by compounds in extracts of Agnus castus: direct evidence for a dopaminergic principle by the dopamine receptor assay. Exp Clin Endocrinol 1994; 102: 448-454

42 Mertz PG, Gorkow C, Schrodter A, Rietbrock S, Sieder C, Loew D, DericksTan JS, Taubert HD. The effects of a special Agnus castus extract (BP1095E1) on prolactin secretion in healthy male subjects. Exp Clin Endocrinol Diabetes 1996; 104: 447-453

43 Dericks-Tan JSE, Schwinn P, Hildt C. Dose-dependent stimulation of melatonin secretion after administration of Agnus castus. Exp Clin Endocrinol Diabetes 2003; 111: 44-46

44 Van Die M, Burger H, Teede H, Bone K. Vitex agnus-castus (Chaste-tree/ berry) in the Treatment of Menopause-related Complaints. J Altern Complement Med 2009; 15: 853-862

45 Diaz BL, Llaneza PC. Endocrine regulation of the course of menopause by oral melatonin: first case report. Menopause 2008; 15: 388-392

46 Jarry H, Spengler B, Porzel A, Schmidt J, Wuttke W, Christoffel V. Evidence for estrogen receptor beta-selective activity of Vitex agnus-castus and isolated flavones. Planta Med 2003; 69: 945-947

47 Whelan AM, Jurgens TM, Naylor H. Herbs, vitamins and minerals in the treatment of premenstrual syndrome: a systematic review. Can J Clin Pharmacol 2009; 16: 407-429

48 Bryant M, Cassidy A, Hill C, Powell J, Talbot D, Dye L. Effect of consumption of soy isoflavones on behavioural, somatic and affective symptoms in women with premenstrual syndrome. Br J Nutr 2005; 93: 731-739

49 Cos P, De Bruyne T, Apers S, Vanden Berghe D, Pieters L, Vlietinck AJ. Phytoestrogens: recent developments. Planta Med 2003; 69: 589-599

50 Bendich $A$. The potential for dietary supplements to reduce premenstrual syndrome (PMS) symptoms. J Am Coll Nutr 2000; 19: 3-12

51 Hagen I, Nesheim BI, Tuntland T. No effect of vitamin B-6 against premenstrual tension. A controlled clinical study. Acta Obstet Gynecol Scand 1985; 64: 667-670

52 Doll H, Brown S, Thurston A, Vessey M. Pyridoxine (vitamin B6) and the premenstrual syndrome: a randomized crossover trial. J R Coll Gen Pract 1989; 39: 364-368

53 Wyatt KM, Dimmock PW, Jones PW, Shaughn O'Brien PM. Efficacy of vitamin B-6 in the treatment of premenstrual syndrome: systematic review. BMJ 1999; 318: 1375-1381

54 Gumm R, Cunnick GH, Mokbel K. Evidence for the management of mastalgia. Curr Med Res Opin 2004; 20: 681-684

55 Mansel RE, Dogliotti L. European multicentre trial of bromocriptine in cyclical mastalgia. Lancet 1990; 335: 190-193

56 Van Die MD, Bone KM, Burger HG, Reece JE, Teede HJ. Effects of a combination of Hypericum perforatum and Vitex agnus-castus on PMS-like symptoms in late-perimenopausal women: findings from a subpopulation analysis. J Altern Complement Med 2009; 15: 1045-1048

57 American Psychiatric Association. Diagnostic and statistical manual of mental disorders, 3rd edition, revised (DSM-III-R). Washington DC: APA; 1987: 715-718 